\title{
Emergency Medicine Training and Practice in Canada: Celebrating the Past \& Evolving for the Future
}

The Collaborative Working Group on the Future of Emergency Medicine in Canada (CWG-EM): Douglas Sinclair, $\mathrm{MD}^{*{ }^{+}}$; Riyad B. Abu-Laban, MD, MHSc ${ }^{\S \uparrow ;}$; Peter Toth, MD, BSc, MSc ${ }^{\neq \|}$; Constance LeBlanc, $\mathrm{MD}^{* *+\dagger}$; Pamela Eisener-Parsche, $\mathrm{MD}^{\ddagger \neq \uparrow \uparrow}$; Jason R. Frank, MD, MA(Ed) $)^{\S \S \| l !}$; Brian Holroyd, $\mathrm{MD}, \mathrm{MBA}^{* * *+\dagger \dagger}$

\section{EXECUTIVE SUMMARY}

Postgraduate Emergency Medicine (EM) training and certification in Canada currently consists of two separate training pathways that are overseen by two autonomous national colleges. The Royal College of Physicians and Surgeons of Canada (Royal College) and the College of Family Physicians of Canada (CFPC) independently offer EM residency training programs with differing training requirements and objectives (FRCPC-EM and CCFP(EM), respectively). Each program was originally intended to fulfill differing societal and healthcare needs. In reality, the products of these training programs significantly overlap, and have evolved to meet population needs differently than their initially intended roles as outlined by the two colleges, leading to substantial debate within the Canadian EM community.

We invite you to view the full report online by visiting one our trilateral partner websites (CAEP, CFPC or Royal College) or by the following link: www.caep.ca/ resources/collaborative-working-group-final-report.

\section{PROJECT PARAMETERS}

The prime objective shared by both the Royal College and the CFPC is to ensure that expert

EM graduates provide high quality EM care for patients presenting to emergency departments (EDs) across Canada. The Collaborative Working Group on the Future of Emergency Medicine in Canada (CWGEM) was constituted in 2013 to provide recommendations to the Canadian Association of Emergency Physicians (CAEP), the CFPC, and the Royal College and was composed of seven (7) members: a Chair, and two (2) members appointed by each of CAEP, the CFPC, and the Royal College. The Terms of Reference of the CWG-EM, including roles and responsibilities, membership, and decision making process, are provided in Appendix A of the final report.

The following report describes the activities, findings, and recommendations of the CWG-EM, with the ultimate goal of outlining a path forward that enhances EM training and care in Canada. Informed by the past and present state of EM training and practice, the CWG-EM report is fundamentally focused on charting a course for the future of emergency medicine in Canada.

\section{PROJECT APPROACH}

The project involved a number of methods to capture and synthesize data for review by the CWG-EM, including:

- An analysis of historic materials, initiatives, and perspectives of the EM community - A review of relevant literature was performed in order to

From * St. Michael's Hospital, Toronto, ON; †Faculty of Medicine and $\ddagger$ Department of Family and Community Medicine, University of Toronto, Toronto, ON, §Department of Emergency Medicine, University of British Columbia, Vancouver, BC, IVancouver General Hospital, Vancouver, BC, $\|$ Credit Valley Hospital, Trillium Health Partners, Mississauga, ON, **Department of Emergency Medicine, Dalhousie University, Halifax, NS, ††QEII Health Sciences Centre, Halifax, NS, ‡¥Department of Family Medicine and §§Department of Emergency Medicine, University of Ottawa, Ottawa, ON, ITBruyère Continuing Care, Ottawa, ON, IIISpecialty Education, Royal College of Physicians and Surgeons of Canada, Ottawa, ON, ***Department of Emergency Medicine, Faculty of Medicine and Dentistry, University of Alberta, Edmonton, AB; and the †††Emergency Strategic Clinical Network, Alberta Health Services, Edmonton, AB.

Correspondence to: Douglas Sinclair, St. Michael's Hospital, 30 Bond Street, Toronto, ON M5B 1W8; Email: sinclaird@smh.ca 
obtain a comprehensive understanding of previously described or published perspectives of the EM community, review the findings of relevant published studies, as well as review past initiatives on these issues.

- A comparative analysis of the CCFP(EM) and FRCPC-EM training routes - A comparison of the program goals, objectives, training pathway, and certification of both routes was completed.

- Development of key project questions - Between October 2013 and February 2014, the CWG-EM developed a series of key questions (Appendix B of the final report) that were aligned with the roles and responsibilities defined by the group's Terms of Reference. The development of these questions, subsequent research, and determination of the limited depth and breadth of information currently available, led to the decision to conduct a nationwide survey of EM training and health human resource (HHR) needs.

- Communication with University Postgraduate Deans of Medical Education - On September 16, 2014, correspondence was distributed to all 17 Canadian Postgraduate Deans of Medical Education, requesting their perspectives on the two independent training streams for EM certification in Canada.

- A national survey of Emergency Medicine training and health human resource (HHR) needs - A survey of CCFP(EM) and FRCPC-EM certified physicians, CCFP physicians with an interest or activity in EM, CCFP(EM) and FRCPC-EM residents, and ED Chiefs was conducted in the summer of 2015. Approval for this survey was obtained from the Dalhousie University Research Ethics Board.

- Additional activities and communication with key stakeholders. During its mandate, the CWG-EM communicated with a number of groups and stakeholders. A list of communications can be found on page 27 of the final report.

\section{KEY FINDINGS FROM THE NATIONAL SURVEY OF EM} TRAINING AND HHR NEEDS

The findings from the national survey of CCFP(EM) and FRCPC-EM certified physicians, CCFP physicians, EM residents from both programs, and ED
Chiefs were critical in determining the current context of Canadian emergency care, perceptions on training routes, training needs, and estimated health human resources needs both currently and in the future.

\section{Practice profiles of physicians currently practicing EM}

- The primary practice settings for physicians with an EM certification are typically large and small urban settings. FRCPC-EM and dual certificants almost exclusively practice EM in large urban academic settings, while the primary practice setting of CCFP (EM) certificants are more varied across large and small urban settings. These results were consistent with the findings from EM residents, with the majority of FRCPC-EM and CCFP(EM) residents indicating a desire to practice full-time EM in an urban setting.

- The proportion of CCFP(EM) certificants with a component of Family Medicine in their practice is extremely low across all ED settings. EM practice makes up the majority of the clinical practice distribution of all physicians with an EM certification.

- CCFP (non-EM) certified physicians are most likely to provide emergency care in rural settings, and make up the large majority of physicians providing emergency care in remote and rural settings.

\section{Alignment of training and practice}

- The majority of respondents who currently practice EM (FRCPC-EM, CCFP(EM), CCFP) feel adequately prepared for clinical practice by their training route.

- The effectiveness rating of the CCFP (non-EM) program for EM practice is more likely to be rated positively in smaller centres.

- There is a strong sentiment amongst certified physicians and ED chiefs outside rural and remote settings, and trainees in both programs, that CCFP (non-EM) training alone is insufficient to gain competencies for the practice of EM. ED chiefs outside rural and remote settings and certified physicians indicated their greatest concern was with regard to CCFP (non-EM) training alone for the practice of EM in larger centres. 
- Qualitative responses of survey respondents indicated that preparation for practice after graduation is dependent on training route and intended practice setting.

\section{Reflections on the current approach to EM certification in Canada}

- Survey respondents identified strengths and challenges of the dual college, dual certification approach for EM residency training. Many respondents advocated a single unified EM training program, however this was not considered feasible at this time by the CWG-EM.

- A qualitative analysis of the survey data revealed four key strengths and four key challenges of the current approach.

o Strengths: The current approach fulfills Canadian needs, is responsive to Canada's vast geography and population distribution and is appropriate for diverse contexts, includes roles for each practitioner type, and both routes produce capable emergency physicians.

o Challenges: The current approach has insufficient content exposure for both training routes, inappropriate length of training programs, inequalities between the certification routes, and misalignment of program goals with practice.

\section{Physician distribution and staffing needs}

- Current physician staffing needs are not fully covered in any ED setting type.

o Dependent on ED setting, approximately $40 \%$ (large urban academic) to $62.5 \%$ (remote) of ED Chiefs from different ED settings indicated that their staffing needs were not fully covered.

o The most significant hours of shortfall were reported in large urban academic (11.3 hours coverage/day/ED short) and large urban nonacademic (15.4 hours coverage/day/ED short) settings.

- The majority of ED Chiefs anticipate an increase in annual patient volume and a critical staffing shortage.
IMPLICATIONS OF FINDINGS

\section{Current shortfall of certified emergency physicians and projected increase in the HHR deficit over the next decade}

An HHR model was constructed by the CWG-EM in order to determine the current and projected shortfall of emergency physicians over the next decade. This concluded there is a current estimated shortfall of 478 emergency physicians in Canada. In the absence of expansion of EM residency training capacity, this shortfall is projected to rise to 1071 emergency physicians by 2020 and to 1518 emergency physicians by 2025. The methodology, limitations, and assumptions of this model can be found on page 66 of the final report.

\section{Misalignment of the intent of the FRCPC-EM and CCFP (EM) training routes and the reality of Emergency Medicine practice for program graduates}

The surveys of physicians with an EM certification and EM residents indicated that a substantial proportion of respondents report discontent regarding the current approach to EM training and care in Canada. This undercurrent of dissatisfaction conveyed by survey respondents appears to arise from a complex multitude of factors that act to divide the EM community. Within a relatively small discipline like EM, this division is pervasive enough to potentially result in animosity between certificant types and may present significant challenges to an effective system of care.

Our survey data suggests concern exists within the EM community that some physicians are placed into a clinical role they are not prepared for upon graduation. Issues regarding preparedness for practice in a variety of settings for both CCFP(EM) and FRCPC-EM graduates appear to stem from a misalignment of the intent of training with the reality of practice.

\section{CCFP(EM) certified physicians}

The CCFP(EM) program currently consists of two years of Family Medicine, followed by one year of training in EM. The short duration of the EM training component creates a challenge for CCFP(EM) residents to meet all of the competency needs for full-time EM practice upon graduation. Qualitative survey responses indicated a need to explore the 
potential of increasing the exposure of CCFP(EM) residents to core EM areas and, given the ultimate career goals of this population, to explore a reduced exposure to clinical Family Medicine in $\operatorname{CCFP}(\mathrm{EM})$ residency programs.

\section{FRCPC-EM certified physicians}

Concerns were raised from survey respondents regarding the five-year duration of the FRCPC-EM program and the limited exposure of residents to aspects of clinical Family Medicine, specifically transitions of care and community care. The survey results suggest graduates of the FRCPC-EM program may benefit from an increased understanding of how the emergency care provided in ED settings integrates into an individual patient's larger continuum of care.

\section{Status of emergency care in rural Canada}

A significant amount of emergency care in rural and remote settings is currently provided by CCFP certified physicians with little specific EM training. The results from the surveys indicated a strong sentiment from the EM community (CCFP(EM) and FRCPC-EM certificants EM residents from both programs, and ED Chiefs from larger centres) that the two year CCFP (non-EM) training program is not sufficient to appropriately prepare physicians for EM practice as a primary discipline.

\section{Preparing for practice: Supporting a standard of care for "patient zero"}

A major consideration that guided the CWG-EM's review of the data and subsequent recommendations is the concept that a central component to the practice of EM is that "patient zero" (the first patient a graduate from a training program cares for post-graduation as an attending physician) merits excellent care in all settings, irrespective of the certification of the care provider. The following set of consensus statements informed the vision and recommendations of the CWG-EM.

\section{Assumptions}

1. Both EM training routes in Canada are high-quality and are effective educationally.

2. Both EM training routes attract high-quality trainees.
3. Canada currently needs a variety of training routes to serve the emergency care of Canadians effectively across all settings.

4. Canada also needs focused rural EM training to effectively meet the needs of patients in this context.

5. The two EM training programs are not identical, due to design, time, educational experiences, emphasis, and duration of training.

6. Differences and distinctions between graduates of the two programs evolve over time, just as every professional changes their scope of practice and depth of knowledge during their career.

\section{Proposed Concepts}

1. The two programs do not and cannot produce identical graduates.

2. There are qualitative and quantifiable differences between graduates of the two programs upon completion of training.

3. CCFP(EM) graduates, due to their Family Medicine (FM) background and competencies, have additional ability in clinical presentations that overlap with ambulatory FM care including but not limited to holistic communication skills, integration with the community, ambulatory psychiatry and obstetrics.

4. FRCPC-EM graduates, due to their longer dedicated training in EM, have additional abilities, including but not limited to: critical care experience, advanced resuscitation skills, advanced toxicology, pediatric EM, research and a higher level of experience with the management of critically ill patients.

5. The qualitative and quantitative differences in \#4 listed above are most evident in the sickest patients and in the context of more complex and unusual emergency patient presentations and conditions.

6. Due to curriculum differences, FRCPC-EMs have additional training in some areas, including EM administration, research, and pre-hospital care.

7. Despite these significant differences, inter-College collaboration in developing competencies to provide care for all common ED presentation is required for all trainees in order to safely meet the needs of 
"patient zero". It is understood that FRCPC-EM program graduates, as EM consultants, will exceed these in some areas. At the same time, the standard of care required to be clinically competent in the provision of $\mathrm{EM}$ care must be equivalent for both CCFP(EM) and FRCPC-EM graduates for ED presentations that are common to the settings in which they work.

8. With experience, the sophistication of EM care will exceed the aforementioned competency minimum for graduates of both EM training programs, however, FRCPC-EM graduates benefit from greater formal education and exposure upon graduation than $\mathrm{CCFP}(\mathrm{EM})$ graduates.

In moving forward, decision-makers must strive to advance approaches that serve both the EM and ED patient communities. The current reality of Canadian EM practice is that graduates of both the CCFP(EM) and FRCPC-EM programs work side by side clinically in a variety of ED settings, most commonly large urban centres. The two colleges have the ability to positively impact patient care by collaboratively developing parallel foundations of training for EM care delivery.

\section{CWG-EM VISION AND RECOMMENDATIONS}

The following recommendations summarize the collective vision of the CWG-EM, and have been generated after careful consideration of the CWGEM's research findings and communications with key stakeholders. The recommendations articulate a series of achievable actions that it is advised the trilateral partners undertake for the advancement of the future of Emergency Medicine training and practice in Canada.

\section{Health human resources shortfall}

There is a current estimated shortfall of 478 emergency physicians in Canada. This deficit is roughly equivalent to the student body size of an entire Canadian medical school. In the absence of expansion of EM residency training capacity, this shortfall is projected to rise to 1071 physicians by 2020 and to 1518 physicians by 2025 (Figure 1).

We recommend that CAEP, the CFPC, and the Royal College work collaboratively to advocate for the significant EM residency training slot expansion necessary to address the large current and projected future shortfall of certified emergency physicians in Canada. CAEP, CFPC and the Royal College are encouraged to work in collaboration in order to address the current and future HHR deficit in Emergency Medicine, consider the right balance of physicians needed to fill this deficit, and advocate for growth in the programs as defined by the types of graduates needed for a variety of ED settings. In addition, in consultation with both colleges, provincial Departments of Health must also work with the postgraduate offices of medical schools within their jurisdictions to increase the number of EM postgraduate training positions in Canada.

In line with its mission to promote the interests of emergency physicians and the specialty of EM in Canada, CAEP must also advocate for the growth of EM certification programs, as well as hold influential groups accountable to move this issue forward.

\section{Alignment of the CFPC and Royal College Emergency Medicine residency training programs}

Patient needs have been met with the current approach to EM certification in Canada; however, some improvements are felt to be necessary to improve

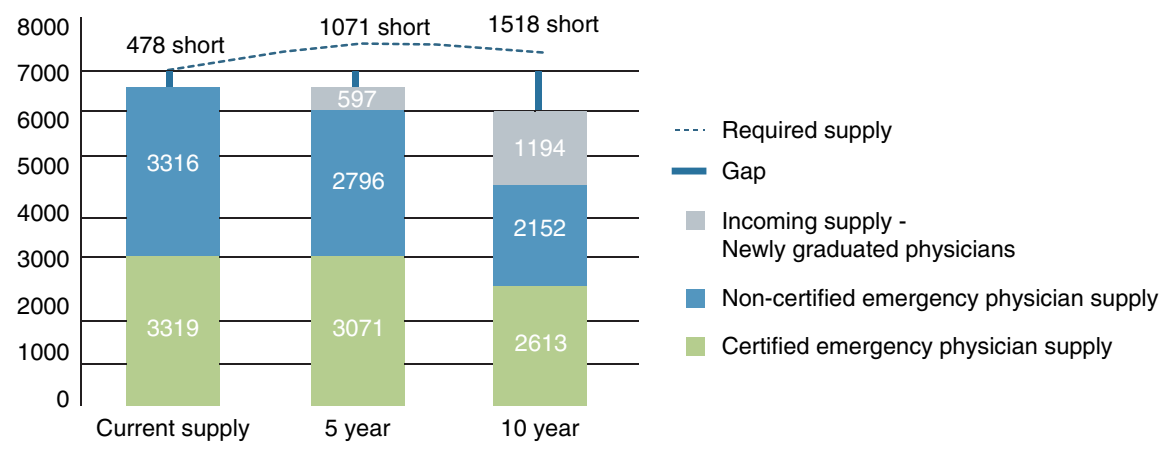

Figure 1. National emergency physician shortfall estimate and future projections (excludes remote settings) 
efficiency and effectiveness and thus enhance care and educational resource utilization. It is envisioned that there will be a continuum of physicians from $\mathrm{CCFP}(\mathrm{EM})$ and FRCPC-EM programs staffing various types of Canadian EDs, with each physician's practice context being aligned with the competencies of the individual.

The CWG-EM is not recommending a single certification stream for EM practice, however the substantial support for this that continues to exist in the EM community underscores the need to make meaningful improvements to the status quo. We recommend that the CCFP(EM) and FRCPC-EM programs reform their objectives of training with the following goals:

\section{FRCPC-EM program}

The focus of the Royal College must be on the development of important competencies to allow graduates to deliver specialist emergency care to patients in tertiary, large urban, regional, and community hospital EDs.

\section{CCFP(EM) program}

Data from the CWG-EM survey of CCFP(EM) certified physicians and residents indicates that the vast majority of graduates of the $\mathrm{CCFP}(\mathrm{EM})$ program practice or intend to practice full-time EM and not a combination of EM and clinical Family Medicine. We recommend that the CFPC focus their efforts on a review of the structure, goals, and objectives of the CCFP(EM) program in order to ensure competency at graduation and to satisfy the standard of care for "patient zero."

- $\operatorname{CCFP}(\mathrm{EM})$ program graduates should have the necessary competencies to deliver emergency care to patients in many contexts including large urban EDs, regional and community hospital EDs, as well as smaller EDs.

- A detailed review of the curriculum of the CCFP (EM) training program from a competency based perspective is required in order to ensure the inclusion of the required competencies necessary to confidently practice full-time EM upon graduation. In the review of the $\mathrm{CCFP}(\mathrm{EM})$ program, it is important to note that while the $\operatorname{CCFP}(\mathrm{EM})$ program likely could continue to be completed in three years, the incorporation of a competency based perspective would almost certainly result in modifications to the traditional two plus one program to an alternative arrangement of program rotations and/or objectives, or alternatively, an increase the program duration for the EM training component.

- Due to the varying needs of different ED locations of CCFP(EM) certificants, some core competencies incorporated into the $\mathrm{CCFP}(\mathrm{EM})$ program should be dictated by the anticipated setting of future practice. The nature of the preceding two years of CCFP training should also be considered at the level of each individual $\mathrm{CCFP}(\mathrm{EM})$ trainee, as inter-program variability exists in the nature of this, particularly regarding whether the focus is rural or urban.

\section{HHR needs in rural and remote settings}

HHR needs in rural and remote areas are complex, and it is unlikely that the full breadth of EM staffing needs can be filled in these locations by CCFP(EM) and FRCPCEM certified physicians in the near future. HHR planning for rural and remote areas needs to consider many more aspects of medical care than solely emergency care. A review of the CCFP (non-EM) program and the issues of EM care in rural Canada were outside of the mandate of the CWG-EM, however general recommendations related to the CCFP program and provision of EM care will be briefly communicated.

The CFPC is encouraged to review the rural/remote findings in detail and, as many CCFP certified physicians fulfill critical ED staffing needs in these settings, consider modifications to the CCFP program in order to ensure that required competencies for provision of emergency care are attained by program graduates intending to work in these settings. We recommend that the CFPC, the Society of Rural Physicians of Canada, and other key stakeholders continue to work collaboratively towards solutions for the provision of optimal emergency care in rural Canada.

\section{Future collaboration of the CCFP(EM) and FRCPC-EM programs}

Collaboration between the two colleges with respect to the CCFP(EM) and FRCPC-EM residency programs must be implemented and actioned towards the goal of achieving clinical competence for every resident, for their ultimate practice trajectory and setting, at the completion of training.

We recommend the two Colleges make specific and meaningful changes to collaborate on issues related to 
their EM training programs and the future evolution of Canadian EM education and certification, including but not limited to:

- Collaboration between the CFPC and the Royal College in order to clarify, co-develop, and distinguish the goals of each program, highlight their distinctions, and ultimately ensure that clinical competencies for "patient zero" are met, non-clinical competencies are achieved, and both programs are efficient and effective at meeting their goals.

- Optimizing patient care by collaboratively developing parallel foundations of EM care for both training routes, as well as the co-development of competencybased clinical care milestones for common clinical presentations.

- Establishing observer status appointments for oneanother on EM relevant committees including but not limited to the Royal College Specialty Committee in Emergency Medicine and the CCFP(EM) Program Committee.

\section{CWG-EM Data Access for Future Research Activities}

The quantitative and qualitative results of the CWGEM National Survey of EM represent a significant collective resource for the Trilateral Partners (CAEP, CFPC, and the Royal College), as well as the Canadian EM community, both today, and in the future.

The opportunity to access the data collected by the CWG-EM should be made available for future research initiatives by any of the trilateral partners or members of the EM research community.

Access to the CWG-EM survey data should be granted exclusively for the purposes of research and improvement to the emergency care of Canadian populations, and not for any commercial purposes. A formal application process should be developed and the CWG-EM should be continued (or a new trilateral partner committee should be established) to review any requests to access raw CWG-EM survey data. Permission to access the CWG-EM survey data would require the approval of all three Trilateral Partner organizations, and approval from a Canadian Research and Ethics Board (REB). Additional factors involving access to CWG-EM survey data such as project mechanics, intellectual property, and confidentiality, would need to be defined in advance by the trilateral partners.
Examples of potential future research questions that could be answered or facilitated through the use of CWG-EM survey data are provided in Figure 23 of the final report.

\section{CONCLUSIONS}

Since the late 1970s, the training in and practice of Emergency Medicine in Canada has undergone significant transformation. Prior to the recognition of EM as a distinct discipline in Canada, EDs were typically staffed by physicians without formal EM training or a comprehensive understanding of the unique requirements of EM practice. During this time, physicians providing care in emergency rooms identified the societal need for a dedicated practice to serve the acutely ill and injured. The formation of CAEP in 1978 and the development of EM residency training programs by the Royal College and the CFPC in the early 1980's have served the emergency care needs of the Canadian public effectively. EM has since evolved into an academic discipline, and the Canadian EM community now has an international reputation of excellence in clinical care, education, and research.

As the discipline of EM continues to evolve and the emergency care needs of the Canadian public become increasingly complex, in part due to an expanding and aging population, the EM community must continue to maintain high expectations for training in and practice of competent care in all emergency settings.

The recommendations made by the CWG-EM are not a judgment on the current variation in practice experience and certification within the EM community of today, but rather represent a collective vision for the future based on findings from the first comprehensive survey of the entire Canadian EM community (including ED chiefs, practicing physicians certified in EM by either the Royal College or the CFPC, and all EM residents in training) as well as other data sources and reviews completed by the CWG-EM.

The CWG-EM has identified a significant HHR shortfall of emergency physicians, both currently and in the future, and has recommended urgent actions be taken to address this issue. In addition, we have also identified a number of practical strategies for both the Royal College and CFPC to enhance and align their EM residency training programs to enhance EM care 
and educational resource utilization. We have recommended that CAEP continue to be an important partner and advocate in the implementation of these recommendations.

This report and its recommendations represent an important contribution to the improvement of EM care for all Canadians by facilitating meaningful changes to EM training and practice. We thank the trilateral partners for their vision in establishing the CWG-EM and urge early engagement with all relevant stakeholders with a goal of implementation of our recommendations over the next year.

Acknowledgements: The CWG-EM acknowledges and extends a special thanks to Ms. Mona Bates, Executive Assistant to the CWG-EM Chair, whose organizational expertise and attention to detail were essential to the success of this project. In addition, the CWG-EM would like to acknowledge the Educational Strategy, Innovations, and Development Unit at the Royal College of Physicians and Surgeons of Canada for its contributions to the analysis and writing of this report on a contractual basis. 